\title{
TRASTORNO DEL ESPECTRO AUTISTA: CARACTERIZACIÓN CLÍNICA EN PACIENTES DE DOS CENTROS DE REFERENCIA EN BOGOTÁ, COLOMBIA
}

\author{
EUGENIA ESPINOSA ${ }^{1}$, PAOLA MERA ${ }^{2}$, DANIEL TOLEDO 3 \\ ${ }^{1}$ Profesor titular universidad Militar nueva granada y universidad del Rosario, \\ ${ }^{2}$ Servicio neurologia infantil Hospital departamental de Nariño \\ ${ }^{3}$ Universidad del Bosque, Bogotá, Colombia. \\ Correspondencia: polyneuroped@gmail.com
}

Recibido: 2 de enero de 2017 Aceptado: 27 de junio de 2017

\section{Resumen}

Introducción. Los pacientes con trastornos del espectro autista (TEA) presentan gran heterogeneidad en sus características comportamentales, cognitivas, médicas y psiquiátricas. En nuestro medio, no existe una descripción de tales variables. Si bien otros estudios han descrito un perfil clínico característico, es necesario conocer nuestra población blanco.

Objetivo. Caracterizar clínicamente los pacientes con diagnóstico de TEA de dos centros de referencia, en Bogotá.

Pacientes y métodos. Estudio retrospectivo observacional y descriptivo de serie de casos documentados en historias clínicas de enero de 2010 a enero de 2014. Se revisaron las historias clínicas de todos los pacientes con TEA confirmada por diagnóstico, incluyendo datos sociodemográficos, antecedentes personales y familiares, así como factores de riesgo descritos en la literatura en relación con la aparición de TEA; ayudas diagnósticas, comorbilidades y tratamiento.

Resultados. Ochenta y un casos cumplieron los criterios de inclusión. En la serie, se encontró predominio en el género masculino $(94,1 \%)$, complicaciones perinatales $(43 \%)$ y prematurez $(7,6 \%)$. Las principales comorbilidades fueron trastornos conductuales (63\%), epilepsia (23,5\%) y trastorno por déficit de atención e hiperactividad (23\%). Fue significativa la asociación entre epilepsia y discapacidad intelectual: $84 \%$ ( $p<0,001)$. La asociación con síndromes genéticos o errores innatos del metabolismo fue mínima en nuestra serie. Los estudios complementarios fueron normales en la mayoría de los casos.

Conclusiones. En nuestra serie predominó el autismo no sindrómico; la presentación clínica, el abordaje diagnóstico y terapéutico concuerdan con lo reportado en la literatura mundial. Los elementos clínicos constituyen la principal herramienta diagnóstica, el manejo conductual es el pilar de tratamiento. Estudios analíticos enfocados hacia las variables más significativas, permitirán la creación de estrategias terapéuticas dirigidas a nuestra población.

Palabras clave: autismo; comorbilidades; comportamiento infantil; factores de riesgo para autismo; trastornos del neurodesarrollo; trastornos pervasivos del desarrollo. 


\title{
AUTISM SPECTRUM DISORDER: CLINICAL CHARACTERIZATION OF PATIENTS AT TWO REFERENCE CENTERS IN BOGOTÁ, COLOMBIA
}

\begin{abstract}
Summary
Introduction: Patients with autism spectrum disorders (ASD) present great heterogeneity in their behavioral, cognitive, medical and psychiatric characteristics. In our environment, there is no description of such variables. Even though other studies have described a characteristic clinical profile, it is necessary to know our white population.
\end{abstract}

Objective: Clinically characterize patients diagnosed with ASD at two reference centers in Bogotá.

Patients and methods: Retrospective, observational and descriptive study of a series of cases documented in medical records from January 2010 to January 2014. The clinical histories of all patients with a confirmed diagnosis of ASD were reviewed, including sociodemographic data, personal and family history, as well as risk factors described in the literature in relation to the occurrence of ASD; diagnostic aids, comorbidities, and treatment.

Results: Eighty-one cases met the inclusion criteria. In the series, predominance was found in the male gender (94.1\%), perinatal complications (43\%) and prematurity (7.6\%). The main comorbidities were behavioral disorders (63\%), epilepsy (23.5\%) and attention deficit hyperactivity disorder (23\%). The association between epilepsy and intellectual disability was significant at $84 \%(\mathrm{p}<0.001)$. The association with genetic syndromes or inborn errors of metabolism was minimal in our series. Complementary studies were normal in most cases.

Conclusions: In our series, non-syndromic autism predominated; the clinical presentation, the diagnostic and therapeutic approach all agree with what is reported in the world literature. The clinical elements constitute the main diagnostic tool, and behavioral management is the pillar of treatment. Analytical studies focused on the most significant variables will allow for the creation of therapeutic strategies aimed at our population.

Keywords: autism; comorbidities; child behavior; risk factors for autism; neurodevelopmental disorders; pervasive development disorders.

\section{TRANSTORNO DO ESPECTRO AUTISTA: CARACTERIZAÇÃO CLÍNICA EM PACIENTES DE DOIS CENTROS DE REFERÊNCIA EM BOGOTÁ, COLÔMBIA}

\section{Resumo}

Introdução. Os pacientes com transtornos do espectro autista (TEA) apresentam grande heterogeneidade em suas características comportamentais, cognitivas, médicas e psiquiátricas. Em nosso meio, não existe uma descrição de tais variáveis. Se bem que outros estudos têm descrito um perfil clínico característico, é necessário conhecer nossa população alvo.

Objetivo. Caracterizar clinicamente os pacientes com diagnóstico de TEA de dois centros de referência em Bogotá.

Pacientes e Métodos. Estudo retrospectivo observacional e descritivo de serie de casos documentados em histórias clínicas de janeiro de 2010 a janeiro de 2014. Revisaram-se as histórias clínicas de todos os pacientes com TEA con- 
firmada por diagnóstico, incluindo dados sócio demográficos, antecedentes pessoais e familiares, assim como fatores de risco descritos na literatura em relação com a aparição de TEA; ajudas diagnósticas, co-morbilidades e tratamento.

Resultados. Oitenta e um casos cumpriram os critérios de inclusão. Na série, se encontrou predomínio no gênero masculino $(94,1 \%)$, complicações perinatais $(43 \%)$ e prematuridade $(7,6 \%)$. As principais co-morbilidades foram transtornos de conduta (63\%), epilepsia (23,5\%) e transtorno por déficit de atenção e hiperatividade (23\%). Foi significativa a associação entre epilepsia e incapacidade intelectual: $84 \%$ ( $p<0,001)$. A associação com síndromes genéticas ou erros inatos do metabolismo foi mínima em nossa série. Os estudos complementares foram normais na maioria dos casos.

Conclusões. Em nossa série predominou o autismo não sindrômico; a apresentação clínica, a abordagem diagnóstica e terapêutica concordam com a informação reportada na literatura mundial. Os elementos clínicos constituem a principal ferramenta diagnóstica, o manejo da conduta é o pilar de tratamento. Estudos analíticos enfocados às variáveis mais significativas permitirão a criação de estratégias terapêuticas dirigidas a nossa população.

Palavras-chave: autismo; co-morbilidades; comportamento infantil; fatores de risco para autismo; transtornos do neuro desenvolvimento; transtornos invasivos do desenvolvimento.

\section{Introducción}

Los pacientes con trastornos del espectro autista (TEA) presentan una importante heterogeneidad en sus características comportamentales, cognitivas, médicas y psiquiátricas $(1,2)$; en el contexto colombiano no existe una descripción de tales eventos, por esta razón, aunque otros estudios ya han mostrado un perfil clínico de estos pacientes, es necesario conocer nuestra población blanco y obtener una visión inicial de la situación del paciente con TEA que acude al Hospital Militar Central (HMC) y al Instituto de Ortopedia Infantil Roosevelt (IOIR), en Bogotá, Colombia. Según lo reportado en la literatura actual, el alcance de las intervenciones terapéuticas con impacto real en el curso de la enfermedad se encuentra aún en desarrollo (3-5). Este estudio pretende generar una base de la que partirán otros estudios analíticos encaminados al desarrollo de estrategias terapéuticas capaces de responder a las circunstancias particulares de nuestra población.

\section{Generalidades de los trastornos del espectro autista}

Los TEA fueron descritos por primera vez en 1940 por Kanner y Asperger (6,7), quienes reportaron de manera independiente sus respectivos grupos de pacientes con los criterios clínicos que siguen vigentes: presencia de intereses y comportamientos restringidos y repetitivos, dificultades con la reciprocidad socioemocional y alteraciones en la comunicación verbal y no verbal $(1,2,8,9)$; estas deficiencias corresponden a los síntomas cardinales del autismo y en el Manual de enfermedades mentales IV (DSM4 por sus siglas en inglés), se consideran criterios diagnósticos siempre que sean persistentes en el tiempo y tengan impacto clínico en la vida del paciente $(2,9)$.

Ante varios esfuerzos por clasificar tales desórdenes, en el DSM4, versión revisada en el 2000, se indicaron 5 posibles diagnósticos dentro del espectro de trastorno pervasivo del desarrollo (2): trastorno autista; síndrome de Asperger; trastorno desintegrativo de la infancia; síndrome de Rett y trastorno pervasivo del desarrollo no especificado, también llamado autismo atípico (2). La quinta edición del Manual de enfermedades mentales (DSM5), si bien conserva los parámetros clásicos mencionados, propone cambios en la manera de agrupar los criterios diagnósticos. Según el manual, en un único ítem A, se encuentran las dificultades en interacción social y las de comunicación (criterios separados en DSM4); considera además que puede realizarse un diagnóstico a una edad más tardía que la indicada en DSM4, según el cual, los sintomas deben haber iniciado antes de los tres años (2) e indica que se deben tener en cuenta las manifestaciones clínicas presentes en el momento de la consulta y las informadas en los antecedentes del paciente $(8,9)$.

El DSM5, además, agrega una clasificación por gravedad de los síntomas, dada por el grado de discapacidad y requerimiento de soporte del paciente $(8,9)$. Los pacientes con trastorno generalizado del desarrollo, autismo y síndrome de Asperger se unifican dentro del grupo de 
TEA y, según el manual, se denominarán así siempre que hayan sido diagnósticados adecuadamente con los criterios del DSM4 (8). Cuando existe TEA asociado a una comorbilidad genética o médica, el DSM5 indica registrar el TEA asociado, nombre de la afección, trastorno o factor que produce tal condición: en el caso de Síndrome de Rett, existe un TEA asociado a síndrome de Rett. La tabla 1 resume los criterios diagnósticos de DSM5.

Tabla 1. Criterios diagnósticos de TEA, según el DSM5*.

A. Deficiencias persistentes en la comunicación social y en la interacción social en diversos contextos, manifiestas por lo siguiente, actualmente o por los antecedentes:

1. Deficiencias en la reciprocidad socioemocional.

2. Deficiencias en las conductas comunicativas no verbales utilizadas en la interacción social.

3. Deficiencias en el desarrollo, mantenimiento y comprensión de las relaciones sociales.

B. Patrones restrictivos y repetitivos de comportamiento, intereses o actividades, que se manifiestan en dos o más de los siguientes puntos, actualmente o por los antecedentes:

1. Movimientos, utilización de objetos o habla estereotipados o repetitivos.

2. Insistencia en la monotonía, excesiva inflexibilidad de rutinas o patrones ritualizados de comportamiento verbal o no verbal.

3. Intereses muy restringidos y fijos que son anormales en cuanto a su intensidad o foco de interés.

4. Hiper- o hiporreactividad a los estímulos sensoriales o interés inhabitual por aspectos sensoriales del entorno.

C. Los síntomas han de estar presentes en las primeras fases del período de desarrollo.

D. Los síntomas causan un deterioro significativo clínicamente en lo social, laboral u otras áreas importantes del funcionamiento habitual.

E. Estas alteraciones no se explican mejor por la discapacidad intelectual o por el retraso global del desarrollo. Si hay comorbilidad con discapacidad intelectual, la comunicación social ha de estar por debajo de lo previsto para el nivel general de desarrollo.

\footnotetext{
La gravedad se clasifica en niveles y se basa en deterioro de la comunicación social y en patrones de comportamiento restringidos y repetitivos: Nivel 1: requiere apoyo. Nivel 2: requiere apoyo sustancial. Nivel 3: requiere apoyo muy sustancial.
}

Ante la modificación de los criterios diagnósticos, establecer una prevalencia real se ha convertido en un reto $(10,11)$; revisiones de estudios realizados en Canadá, USA, Japón, Alemania y Francia, estiman una prevalencia de 10-27,2 en 10 mil niños con edades entre los 3-10 años (12-14). Estas cifras varían, algunos autores han encontrado prevalencias de $1-10$ por cada 10000 , dependiendo de los métodos y diseños de estudio $(5,13-$ 15). Otros estudios, realizados a través del Autism and Developmental Disabilities Monitoring (ADDM), han mostrado un aumento del $78 \%$ en la prevalencia, comparando estudios de 2002, en los que se encuentra prevalencia de 6,4, por cada 1000 niños, frente a estudios de 2006, en los que la prevalencia es de 9,0 también por cada 1000 (13). En contraste, en Colombia no hay datos epidemiológicos reportados (16).

En relación con el género, Kanner y Asperger observaron que se trataba de un desorden casi exclusivo de los varones $(6,7)$, lo que se confirma con estudios que reportan una frecuencia cuatro veces menor en mujeres (11). Según datos emitidos por el American College of Medical Genetics, se presenta con mayor frecuencia entre hermanos. Por su parte, las Guías prácticas de Genómica sugieren que cuando hay diagnóstico de autismo típico en una mujer, la probabilidad de que otro hermano lo padezca aumenta a un 7,0\%; si en cambio el afectado es un varón, esta cifra será de un $4 \%$ (17). Finalmente, el riesgo de TEA puede alcanzar un $30 \%$ cuando hay dos o más niños afectados en la misma familia $(12,17)$.

En relación con los factores de riesgo, se han propuesto múltiples condiciones, tales como edad y nivel educativo parental $(12,18)$. Se ha reportado que la edad parental puede ser un factor causal independiente, que incrementa el riesgo por cada 10 años que la edad paterna y materna aumenten $(12,18)$. El nivel educativo parental puede influir, pero esta asociación aún no está bien determinada $(5,12)$; la privación ambiental puede agravar la severidad de un TEA subyacente, más que ser el factor causal (5). El diagnóstico de TEA en padres de niños afectados puede ser posible hasta en un $15 \%$ (19), se ha encontrado que muchos de los padres de estos niños tienen una capacidad de respuesta $e$ interacción social deficiente con respecto a padres de niños sanos $(19,20)$.

La prematurez, las gestaciones múltiples y el bajo peso al nacer son factores fuertemente asociados con el desarrollo tardío de autismo $(12,21,22)$. La exposición prenatal 
a rubeola congénita se ha encontrado en el $0,75 \%$ de pacientes con TEA $(12,23)$. También se ha reportado como factor de riesgo la ingesta durante la gestación de fármacos como ácido valproico, etanol, talidomida, misoprostol y tabaco $(21,24-26)$.

La existencia de comorbilidades asociadas es una condición frecuente (12), se encuentran reportados trastornos neuropsiquiátricos como TDAH, desórdenes de ansiedad, problemas conductuales, depresión, retraso del neurodesarrollo y discapacidad intelectual.

Se ha descrito asociación con síndromes genéticos como $\mathrm{X}$ frágil, neurofibromatosis y esclerosis tuberosa entre otros. Sin embargo, lo usual es que se trate de una entidad no sindrómica $(1,12)$.

La comorbilidad con epilepsia varía del $6 \%$ al $30 \%$ y será más frecuente cuando se acompaña de discapacidad intelectual $(\mathrm{IQ}<70)$, con cifras de 21,5-70\% $(27,28,29)$. Las comorbilidades psiquiátricas y neurológicas son más prevalentes y severas en el género femenino $(12,29,30)$.

Otro aspecto relevante es la amplia discusión del modelo etiológico, del cual existen múltiples teorías: origen genético, ambiental, noxa perinatal, infecciones prenatales exposición prenatal a fármacos y tóxicos y exposición a vacunas; ninguna de estas alternativas ha sido probada de manera concluyente. En cuanto al modelo fisiopatológico, se han planteado las teorías de neuronas en espejo, de la mente, alteración funcional de neurotransmisores y alteraciones estructurales en el cerebro social; sin llegar tampoco a una conclusión definitiva (31-39).

Las anomalías en genes o cromosomas pueden representar el 10-41\% de los pacientes con autismo, dependiendo del diseño del estudio $(1,17,40)$; existen escasas alteraciones cromosómicas claramente asociadas a TEA, entre ellas el síndrome Angelman, el síndrome de Williams (duplicación 7q11.23), duplicación o deleción $16 p 11.2$ (40). Se puede encontrar en trastornos monogénicos $(17,40)$ : neurofibromatosis tipo 1 en un $0,3 \%$ y esclerosis tuberosa en un $45 \%$. El síndrome de cromosoma X frágil constituye el 2,7\% de niños con autismo (40).

En el presente estudio se tuvieron en cuenta los factores de riesgo y modelos etiológicos anotados, así como la descripción de otras comorbilidades reportadas en la literatura, tales como síntomas gastrointestinales, trastor- nos del sueño, problemas alimentarios, dificultades con autocuidado, trastornos de conducta y ansiedad (41-45).

En la actualidad, el diagnóstico sigue siendo netamente clínico y se realiza con el cumplimiento de los criterios descritos $(1-3,9)$. Además, existen instrumentos complementarios dirigidos a evaluar y objetivar la sospecha diagnóstica, pero todos requieren profesionales con amplia experiencia en la aplicación e interpretación de resultados $(46,47)$.

El manejo terapéutico continúa siendo de apoyo y las principales intervenciones terapéuticas reportadas tienen su fundamento en el área conductual, con el fin de mejorar la comunicación e integración del individuo en sociedad (3,48-51). Las guías internacionales recomiendan no intentar modular los sintomas nucleares del TEA mediante tratamientos con antipsicóticos, antidepresivos, anticonvulsivantes o dietas restrictivas (libres de gluten, cafeína y caseína) $(3,17)$. La evidencia señala que aún no existen tratamientos farmacológicos convencionales ni terapias alternativas que sean curativas (3, 48-51).

\section{Metodología}

Estudio retrospectivo descriptivo de serie de casos de pacientes con diagnóstico de TEA. De la base de datos de pacientes con diagnóstico de TEA atendidos en la consulta ambulatoria de Neuropediatría y Psiquiatría Infantil del HMC y el IOIR, se obtuvo un total de 130 historias clínicas, cuyo seguimiento corroboró el diagnóstico de autismo por los propios tratantes o la junta de observación de autismo. Del análisis de las 130 historias clínicas, se concluyó que 81 pacientes podían ingresar al protocolo, pues cumplían con todos los criterios de inclusión; la exclusión de los otros 49 pacientes obedeció a que, si bien cumplían los criterios diagnósticos, en las respectivas historias clínicas no estaba registrada gran parte de los datos requeridos en el instrumento de recolección de información ni el seguimiento que habían tenido.

La base de datos se construyó a partir de la información consignada en las historias clínicas, las cuales se ubicaron por número de identificación para proteger la identidad de los pacientes. Se extrajeron datos demográficos, historia perinatal, comorbilidades, antecedentes personales, familiares y tratamientos recibidos. Dado que no se hizo intervención directa con ningún paciente y se protegió su identidad, no se exige consentimiento informado, debido a que la Resolución №8430 de 1993 de ministerio de protección social de Colombia lo permite en tales ca- 
sos. Además, la investigación contó con la aprobación del Comité de Ética del HMC y el IOIR y los resultados fueron llevados a estadística para realizar una descripción de los hallazgos y hacer correlación con lo hallado en la literatura.

Los Criterios de inclusión fueron pacientes con TEA que asistieron a consulta de Neurología Pediátrica de enero de 2010 a enero de 2014 en el HMC y el IOIR. El diagnóstico fue realizado por los servicios de Neurología Pediátrica, Psiquiatría Infantil o la Junta de TEA en el IOIR y cumplen los criterios diagnósticos establecidos por los DSM 4 y 5.

Dentro de los Criterios de exclusión, se encuentran síndromes genéticos identificados que cursen con elementos autistas y no con TEA bien definido por los criterios establecidos; pacientes cuyas alteraciones en su nivel de comunicación pueden explicarse por padecer condiciones tales como parálisis cerebral o discapacidad intelectual grave. Se excluyó de la investigación aquellas historias clínicas en las que no se encontró concordancia con los criterios diagnósticos establecidos por los DSM 4 y 5 o en las que la información era insuficiente.

Para el análisis de datos, se emplearon medidas de tendencia central en variables de tipo numérico; para las variables categóricas se describen tablas de frecuencias y porcentajes absolutos y validados. Para los análisis se utilizó el paquete estadístico SPSS (v 23.0) licenciado por la UMNG.

\section{Resultados}

Se obtuvo una población total de 81 niños $(\mathrm{N}=81)$, de los cuales $8,6 \%$ fueron de género femenino y $91,4 \%$ masculino; en un rango de edades entre los 2 y los 19 años, con una media de edad de 7,6 años al momento de la consulta. La mayoría de las familias procedían de Bogotá y en menor frecuencia de ciudades y municipios aledaños.

En cuanto a antecedentes familiares, la media de edad de la madre al momento del parto fue de 28 años, con un rango de 15-46 años y la del padre de 32,4 años, con un rango de edades de 21-55 años. El 23,5\% de los casos tenía algún familiar afectado con diferentes enfermedades psiquiátricas (esquizofrenia, depresión, trastorno afectivo bipolar, entre otros). Sin embargo, no hubo predominio de ninguna entidad en particular; en el $6,2 \%$ se encontró un familiar afectado con TEA o un hermano en el $9,9 \%$. El porcentaje de consanguinidad parental fue $1,2 \%$. Finalmente, el $29 \%$ no tenía reportado este dato en la historia clínica.

El 5\% de los casos fueron embarazos gemelares. No se reportaron casos STORCH, ni exposición a tóxicos ingeridos en periodo perinatal. El $43 \%$ de las madres presentó algún tipo complicación en el periodo perinatal: preeclampsia en $1,2 \%$, anormalidades placentarias $2,5 \%$, e infección de las vías urinarias o corioamnionitis en un $2,5 \%$. Se documentó sufrimiento fetal agudo en $9,9 \%$ de los pacientes. La mediana de la edad gestacional al momento del parto fue de 38 semanas, el porcentaje de prematuros en toda la serie fue $8,6 \%$ : moderadostardíos $4,9 \%$ y muy prematuros de $2,7 \%$, no se encontró en esta serie prematuros extremos, de acuerdo con la definición de la Organización Mundial de la Salud (OMS).

El APGAR a los 5 minutos fue mayor de 6 en el 80,2\% de los casos, de esta serie el $6,2 \%$ requirió ventilación mecánica durante el periodo neonatal.

El peso al nacer presentó una distribución normal con una media de 3027 gramos, con peso mínimo reportado de 850 gramos y máximo de 4250 gramos El 2,5\% presentó restricción del crecimiento intrauterino de causa no determinada.

La media de edad a la que los niños acudieron a su primera consulta con neurología infantil fue de 7 años; la edad a la que se hizo el diagnóstico de TEA fue en promedio 5 años.

El neurodesarrollo fue adecuado en el $42 \%$ de pacientes durante el primer año de vida; en el segundo año el $75 \%$ ya presentaban alteraciones, de los cuales el $51 \%$ presentaba compromiso mixto del desarrollo; $11 \%$ alteraciones exclusivamente motoras y un $22 \%$ confinado al área del lenguaje. En el tercer año el perfil de TEA estaba bien establecido en el $82 \%$ de los casos, de los cuales el compromiso limitado al área del lenguaje ascendió a un $50 \%$, compromiso mixto baja a $33 \%$ y no se registran trastornos exclusivamente motores.

El lenguaje fue verbal en $49 \%$, pero con un contenido no evaluable en un 76,5\% de ellos. El nivel de compromiso en habilidad social, según lo establecido por el DSM5, fue severo en $39,5 \%$, moderado $34,6 \%$ y leve en $19,8 \%$. 
Se realizó resonancia magnética cerebral en el 44,4\% de pacientes, de estos el $86 \%$ fueron normales.

Los potenciales evocados auditivos fueron realizados en el 34,6\% y fueron anormales en el 1,2\%. El electroencefalograma se realizó en $28 \%$ de niños, con resultados de anormalidad en 9,9\%.

La comorbilidad con epilepsia estuvo presente en $23,5 \%$ de los pacientes y un $4,9 \%$ requirió terapia múltiple para el control de las crisis debido a la refractariedad.

La coexistencia de epilepsia y TDAH ocurrió en el 6,2\%. El TDAH como comorbilidad aislada se presentó en un $23,5 \%$. Los manejos instaurados para el TDAH fueron conductuales en un $19 \%$ y farmacológico en $9,9 \%$ (metilfenidato). Un $43 \%$ de pacientes presentó comorbilidad con trastornos de ansiedad. Los trastornos conductuales se presentaron en $63 \%$ de la serie, de ellos un $68 \%$ recibió manejo con risperidona solamente. Los restantes requirieron combinaciones de risperidona e inhibidores de recaptación de serotonina, olanzapina, aripiprazol o haloperidol.

Se encontró discapacidad intelectual en el $79 \%$ de los casos, de estos un $64 \%$ tenía compromiso verbal importante que no permitió realizar pruebas de coeficiente intelectual convencionales. Además, vale tener en cuenta que las pruebas de evaluación intelectual no verbal no estaban disponibles en todos los sitios de donde los pacientes venían remitidos, pues no es un acto de rutina realizarlos en nuestro país, por esta razón este elementó no se incluyó en el estudio.

La toma de cariotipo había sido reportada en 16 casos de nuestra serie $(19,7 \%)$, de los cuales 3 fueron anormales (3,7\%). La coexistencia con un síndrome genético (dos casos de X frágil, uno con Angelman, uno con deleción de 22q11 y uno con cromosoma 22 en anillo) correspondió al $12 \%$ de la serie, pero no se encontró registros de cuáles fueron las pruebas confirmatorias de los casos. En cuanto a errores innatos del metabolismo, se documentó un caso de fenilcetonuria y otro de aciduria glutárica, confirmados por clínica y pruebas cualitativas y cuantitativas de aminoácidos y ácidos orgánicos. Estos casos representan el 2,5\% de la serie. El autismo no sindrómico fue predominante en nuestro estudio.

Los datos sobre hábitos alimentarios aparecieron registrados en el $60 \%$ de los pacientes, un $17 \%$ reportó rigi- dez e inflexibilidad ante introducción de alimentos nuevos y marcada selectividad. Los trastornos de sueño estuvieron presentes en un $19 \%$ de la serie y consistieron en escasas horas de sueño y dificultades para su conciliación. Tales casos fueron tratados con higiene del sueño (50\%), melatonina (25\%) y antihistamínicos (18\%).

La mayoría de los pacientes requería algún grado de soporte en su autocuidado (70\%), especialmente por mal uso de los elementos de aseo. El manejo terapéutico, en la mayoría de los pacientes $(80 \%)$ consistió en terapia conductual (terapias integrales: ocupacional, lenguaje, psicología y física cuando había alteraciones motoras), un $8 \%$ recibió terapia $\mathrm{ABA}$ y el $3,7 \%$ admitió haber intentado al menos una terapia alternativa como restricción de gluten, uso de animales, hidroterapia o musicoterapia. La falta de continuidad en el tratamiento integral se observó en el $72 \%$ de las historias clínicas.

\section{Discusión}

En este estudio se han descrito las principales características encontradas en cuanto a factores de riesgo, comorbilidades y tratamientos relacionados con los TEA en dos centros de remisión de la ciudad de Bogotá; la mayoría de los datos corresponde con lo revisado en la literatura médica. Se observó mayor prevalencia en varones, con una proporción de 8 hombres por cada niña afectada con la patología.

Debe tenerse en cuenta que el tamaño de la muestra es moderado y que los centros donde se realiza el estudio son de referencia, donde son predominantes los casos más complejos, hechos que podrían afectar estas cifras.

El rango de edad al momento de la consulta fue de 2 a 19 años; si bien los pacientes de mayor edad venían con seguimiento de instituciones de menor complejidad, este dato debe incentivar la educación que se provee al personal de salud, para captar pacientes con síntomas de alarma tan tempranos como los del primer año de vida.

En la literatura mundial, la edad parental aparece como un factor asociado a la aparición de autismo y en estudios recientes ha cobrado importancia la edad paterna avanzada $(12,18)$. En nuestro estudio la edad materna tuvo un promedio de 28 años, siendo la máxima 45 años y mínima de 15. La edad paterna máxima fue de 55 años, con un promedio de 32 años, acorde con lo reportado $(12,18)$. 
Se ha comentado que los TEA seguramente tienen una base genética $(12,17,40)$, en nuestro estudio fue baja la proporción de consanguinidad parental. Sin embargo, debe tenerse en cuenta que este dato no estaba reportado en un porcentaje importante de las historias clínicas, hecho que podría modificar los resultados. Un $6,6 \%$ de pacientes tenía algún familiar afectado con TEA y un 9,0\% un hermano. Estas cifras son similares a las probabilidades reportadas en la literatura mundial. Se encontraron solo dos casos de fragilidad del cromosoma $\mathrm{X}$, un caso de síndrome de Angelman, del 22q11 y cromosoma 22 en anillo; dado que la asociación a un síndrome genético o a un error innato del metabolismo fue baja, el único paraclínico obligatorio para el TEA sería determinar fragilidad de cromosoma $\mathrm{X}$ o alteraciones en gen MECP2 solo si la presentación clínica lo amerita, tal como lo sugiere la literatura actual $(17,40)$.

Sigue siendo un tema de investigación importante la forma en que interactúan los factores genéticos y epigenéticos para originar los TEA $(17,40)$. En nuestra serie los factores ambientales estuvieron presentes en casi la mitad de los pacientes: un $43 \%$ presentaron alguna complicación perinatal, pero ninguna de ellas con una cifra tan significativa que implique causalidad; se documentó prematurez en un $8,6 \%$ de la serie, la literatura reporta que la asociación a este factor de riesgo es cada vez más sólida $(12,21,22)$. No se encontró una relación clara con las gestaciones múltiples como lo han reportado otros estudios $(12,21,22)$. La literatura informa sobre exposición a rubeola congénita o fármacos tóxicos ingeridos por la madre durante la gestación $(12,21,23)$, en este estudio no se evidenció la relación de infección perinatal ni exposición a tóxicos.

Un pequeño número de pacientes presentó baja puntuación APGAR, recuperado en todos los casos; sin requerir hospitalizaciones ni intervenciones agresivas en el periodo neonatal.

Los antecedentes neuropsiquiátricos estuvieron presentes en un número importante de familiares de afectados, pero no hubo predominio de ninguna entidad en particular; cabe anotar que, en nuestro medio, no es un acto de rutina interrogar por sintomas de deficiente interacción social en padres, los cuales pueden estar presentes en un número significativo de padres de afectados con autismo $(19,20)$.

El comportamiento del neurodesarrollo fue llamativo en esta serie de casos; un buen número de pacientes presentaba desarrollo motor y cognitivo adecuado para la edad en el primer año. A partir de esta etapa, en la mayoría de las historias se reporta un estancamiento en los hitos motores e incluso regresión en hitos del lenguaje, hallazgos que ya han sido reportados en la literatura $(1,2,9)$. Los pacientes que presentaban un compromiso mixto (lenguaje y motor) desde el inicio de su seguimiento, reportaron en sus historias clínicas una normalización de los hitos motores gruesos después de los 36 meses, no obstante, las habilidades del lenguaje y desempeño social siguieron comprometidas, y todos los criterios diagnósticos de TEA afloraron hasta el momento de su consulta. Vale aclarar que la literatura ha descrito este tipo de evolución (1).

No se encontró correlación entre los hallazgos anormales en las imágenes de resonancia magnética cerebral y aparición de TEA. Se debe anotar que a menos de la mitad de la serie se le realizó este estudio y, de ellos, un número casi despreciable tuvo hallazgos anormales, sin embargo, ya otros estudios han evidenciado la baja prevalencia de anormalidades en las neuroimágenes en pacientes con TEA y asimetrías no significativas que pueden estar en pacientes sanos (52).

La comorbilidad de TEA con epilepsia está reportada en cerca de $30 \%$ de casos $(28,29)$, en nuestra serie un $23 \%$ presentó esta comorbilidad, la mayoría con adecuada respuesta a monoterapia. En cuanto a la relación entre aparición de epilepsia, discapacidad intelectual y autismo, con las pruebas de chi cuadrado, se encontró una asociación estadísticamente significativa en nuestro estudio $(p=0,001)$.

Se ha descrito una asociación importante entre autismo, epilepsia y TDAH en comorbilidad $(28,29)$, sin embargo, esta relación no tuvo relevancia estadística en el presente estudio.

En nuestra serie los trastornos de ansiedad fueron muy prevalentes, tal como reporta la literatura (42-45); los trastornos conductuales que cursan con auto- $y$ heteroagresión se presentaron de manera notable (63\%) y se ha usado risperidona como manejo de primera línea. Es limitado el número de casos en que se logra control adecuado con manejo conductual aislado y otros medicamentos moduladores como anticonvulsivantes a dosis bajas, inhibidores de recaptación de serotonina, haloperidol o aripiprazol; estos últimos, se han usado en una muy baja proporción de pacientes (48-51). 
Se han descrito también alteraciones en patrones alimentarios en un pequeño porcentaje de esta serie, tales como selectividad y rechazo a ciertas texturas; es posible que la cifra obtenida sea más alta, ya que un $40 \%$ de pacientes no tenía datos registrados al respecto.

Los trastornos del sueño por dificultades de conciliación $y$ ciclos cortos tienen una prevalencia importante en este grupo y la primera línea de manejo es la higiene del sueño y melatonina en concordancia con lo reportado por otros estudios $(3,42-45)$. El autocuidado y la independencia de estos pacientes para sus labores cotidianas están comprometidos casi en todos los casos en diferentes grados de gravedad.

Siguiendo los lineamientos de las guías internacionales (48-51), las intervenciones terapéuticas encontradas en la mayoría de las historias clínicas consistieron en el manejo conductual interdisciplinario: psicología, terapia del lenguaje, ocupacional y física cuando el caso lo requiere. En un número menor de pacientes se describe específicamente la terapia ABA. Un factor que resulta preocupante es que en la mayoría de los casos no existe una continuidad en el tratamiento debido a problemas del sistema de salud; los pocos casos en que las intervenciones se han garantizado de manera continua corresponden a pacientes del hospital Militar Central, donde las fuerzas militares garantizan planes terapéuticos que se renuevan cada año bajo la directriz del especialista tratante.

Dentro de las limitaciones del estudio, podemos describir que se trata de un estudio exploratorio, sin una hipótesis previa; los resultados, aunque en parte han sido similares a la literatura, no se pueden extrapolar a la población pediátrica en general. Se requieren estudios con intervención directa con el paciente y cuidadores, que permita valorar la funcionalidad del niño, detallar más el tratamiento que recibe y la evolución percibida por los padres ante las intervenciones terapéuticas instauradas.

\section{Conclusiones y recomendaciones}

Los TEA cursan con gran heterogeneidad en su presentación clínica, los múltiples factores ambientales y comorbilidades asociados hacen que el diagnóstico oportuno se convierta en un reto. Sigue primando la clínica del paciente y los criterios diagnósticos son la clave para identificar estas entidades. Por esto, es necesario sensibilizar al personal de salud acerca de signos de alarma tempranos. Las ayudas diagnósticas como neuroimáge- nes y pruebas neurofisiológicas, genéticas o metabólicas constituyen una herramienta secundaria en el momento de realizar el diagnóstico y deben solicitarse solo si hay una sospecha clínica fundamentada de un trastorno subyacente. El pilar de tratamiento sigue siendo el manejo conductual y el control oportuno de las comorbilidades más frecuentes.

El abordaje interdisciplinario aumenta la probabilidad de un diagnóstico correcto y un seguimiento terapéutico eficaz, donde las especialidades implicadas se retroalimentan y realizan acuerdos sobre las estrategias más adecuadas para cada paciente. Es de importancia mencionar que cuando los pacientes cuentan con un sistema de apoyo que involucra las áreas sociales, asistenciales y económicas, se facilita la continuidad del tratamiento y seguimiento.

Este es el primer estudio descriptivo sobre TEA realizado en Colombia y con sus hallazgos se ha logrado disponer de una información que servirá como base para posteriores estudios analíticos.

\section{Responsabilidades éticas}

Confidencialidad de los datos: los autores declaran que han seguido los protocolos de su centro de trabajo sobre la publicación de datos de pacientes. Derecho a la privacidad y consentimiento informado: no fue necesario el consentimiento informado debido a que se trató de un estudio sin intervención directa y con protección de la identidad del paciente, lo cual está autorizado por la Resolución № 8430 de 1993 del Ministerio de Protección Social de Colombia.

\section{Conflicto de intereses}

Los autores declaran no tener algún conflicto de intereses.

\section{Agradecimientos}

Dra. Yuly Guzmán. Médica y Epidemióloga.

\section{Referencias}

1. Deborah G. Hirtz, Ann Wagner, Pauline A. Filipek. Chapter 48, part VII, Neurodevelopmental disorders -Autistic spectrum disorders. En: Swaiman K, Ashwal S, Ferriero D, 
Schor N, editors. Swaiman's Pediatric Neurology. 5th ed. Saunders Elsevier; 2012. Pp. 638-63.

2. Asociación Americana de Psiquiatría, Versión española de la cuarta edición de la obra original en lengua inglesa Diagnostic and Statistical Manual of Mental Disorders: DSM-IV, publicada por la American Psychiatric Association de.Washington, DC 1994. Ed MASSON, Barcelona; 1995. Pp. 69-82.

3. National Institute for Health and Care Excellence. Autism: The management and support of children and young people on the autism spectrum. NICE; 2013. Pp.11-26.

4. Amaia Hervás, Luis Sánchez Santos, Autismo - Espectro autista. Del Curso de psiquiatría infantil, Sociedad española de pediatría extrahospitalaria y atención primaria; 2014. Consultado: agosto de 2015. Disponible en: http://www. sepeap.org/wpcontent/uploads/2014/02/Ps_inf_autismo_espectro_autista.pdf

5. Kogan MD, Blumberg SJ et al. Prevalence of parent-reported diagnosis of autism spectrum disorder among children in the US. Pediatrics, 2007 124: 1395-1403 6. Kanner L. (1943). Autistic disturbances of affective contact. Acta Paedopsychiatr. 1968; 35(4): 100-36.

7. Asperger H. Die "Autistischen Psychopathen" im Kindesalter. Archiv für Psychiatrie und Nervenkrankheiten. 1944; 117(1): 76-136.

8. Nazeer A, Ghaziuddin M. Autism Spectrum Disorders: Clinical features and diagnosis. Pediatr Clin N Am. 2012; 59: 19-25.

9. Asociación Americana de Psiquiatría, Guía de consulta de los criterios diagnósticos del DSM 5. Arlington, VA, Asociación Americana de Psiquiatría, 2013. Pp. 28-33.

10. Mattson J, Kozlowski M. The increasing prevalence of autism spectrum disorders. Research in Autism Spectrum Disorders. 2005; 5: 418-25.

11. Chakrabarti S, Fombonne E. Pervasive developmental disorders in preschool children: Confirmation of high prevalence. A J of Psychiatry. 2005; 162: 1133-41.

12. Duchan E, Patel R. Epidemiology of Autism Spectrum Disorders. Pediatr Clin N Am. 2012; 59: 27-43.

13. Kulage KM, Smaldone M, Cohn G. How Will DSM-5 Affect Autism Diagnosis? A Systematic Literature Review and Meta-analysis. J Autism Dev Disord. 2014; 44: 191832.

14. Honda H, Shimizu Y, Imai M, Nitto Y. Cumulative incidence of childhood autism: A total population study of better accuracy and precision. Dev Med and Child Neurol. 2005; 47(1): 10-18.

15. Baxter A, Brugha T, Erskine H, Scheurr R, Vos T, Scott J. The epidemiology and global burden of autism spectrum disorders. Psychol Med. 2015; 45(3): 601-13.

16. Talero C. Autismo: estado del arte. Rev. Cienc. Salud / Bogotá (Colombia). 2003; 1(1): 68-85.

17. Schaefer G, Mendelsohn N. Clinical genetics evaluation in identifying the etiology of autism spectrum disorders: 2013 guideline revisions. Genet Med 2013; 15: 399-407.
18. Croen L, Najjar D. Maternal and paternal age and risk of autism spectrum disorders. Arch Pediatr Adolesc Med. 2007; 161(4): 334-40.

19. Scheeren A, Stauder J. Broader autism phenotype in parents of autistic children: reality or myth? J Autism Dev Disord 2008; 38(2): 276-87.

20. Cederlund M, Gillberg C. One hundred males with Asperger syndrome: a clinical study of background and associated factors. Dev Med Child Neurol. 2004; 46(10): 652-60.

21. Hultman C, Sparen P, Cnattingius S. Perinatal risk factors for infantile autism. Epidemiology. 2002; 13(4): 417-23.

22. Williams K, Helmer M, Duncan G, et al. Perinatal and maternal risk factors for autism spectrum disorders in new South Wales, Australia. Child Care Health Dev 2008; 34(2): 249-56.

23. Fombonne E. The epidemiology of autism: a review. Psychol Med 1999; 29(4): 769-86.

24. Dufour-Rainfray D, Vourc'h P, Tourlet S, et al. Fetal exposure to teratogens: evidence of genes involved in autism. Neurosci Biobehav Rev 2011; 35(5): 1254-65.

25. Stromland K, Nordin V, Miller M, et al. Autism in thalidomide embryopathy: a population study. Dev Med Child Neurol 1994; 36(4): 351-6.

26. Schmidt R, Hansen R, Hartiala J, et al. Prenatal vitamins, one-carbon metabolism gene variants, and risk for autism. Epidemiology 2011; 22(4): 476-85.

27. De Jong M, Punt M, De Groot E, et al. Minor neurological dysfunction in children with autism spectrum disorder. Dev Med Child Neurol. 2011; 53(7): 641-6.

28. Danielsson S, Gillberg I, Billstedt E, et al. Epilepsy in young adults with autism: a prospective population-based follow-up study of 120 individuals diagnosed in childhood. Epilsepsia 2005; 46(6): 918-23.

29. Amiet C, Gourfinkel-An I, Bouzamondo A, et al. Epilepsy in autism is associated with intellectual disability and gender: evidence from meta-analysis. Biol Psychiatry 2008; 64(7): 577-82.

30. Tuchman R, Rapin I, Shinnar S. Autistic and dysphasic children. I: clinical characteristics. Pediatrics 1991; 88(6): 1211-8.

31. Iacoboni M, Dapretto M. The mirror neuron system and the consequences of its dysfunction. Nature Reviews Neuroscience. 2006; 7: 942-51.

32. Conturo T, Williams D, Smith C, Gultepe E, et al. Neuronal fiber pathway abnormalities in autism: An initial MRI diffusion tensor tracking study of hippocampo-fusiform and amygdalo-fusiform pathways. Journal of the International Neuropsychological Society. 2008; 14: 933-46.

33. Kana R, Keller T, Cherkassky V, Minshew N, Just M. Atypical frontal-posterior synchronization of Theory of Mind regions in autism during mental state attribution. Social Neuroscience. 2009; 4: 135-152.

34. Depue R, Morrone-Strupinsky J. A neurobehavioral model of affiliative bonding: implications for conceptualizing a human trait of affiliation. The Behavioral and Brain Sciences. 2005; 28 (3): 313-350. 
35. Hollander E, Bartz J, Chaplin W, Phillips A, et al. Oxytocin increases retention of social cognition in autism. Biological Psychiatry. 2007; 61: 498-503.

36. Dillon J, Raleigh M, McGuire M, Bergin-Pollack D, Yuwiler A. Plasma catecholamines and social behavior in male vervet monkeys (Cercopithecus aethiops sabaeus). Physiology \& Behavior. 1992; 51 (5): 973-7.

37. Tse W, Bond A. Reboxetine promotes social bonding in healthy volunteers. Division of Psychological Medicine, Institute of Psychiatry, King's College London, UK. Journal of Psychopharmacology. 2003; 17(2): 189-95.

38. Whitaker-Azmitia P. Serotonin and brain development: Role in human developmental diseases. Brain Research Bulletin. 2001; 56(5): 479-85.

39. Anderson G, Freedman D, Cohen D, Volkmar F, et al. Whole blood serotonin in autistic and normal subjects. Journal of Child Psychology and Psychiatry. 1987. 28 (6): 885-900.

40. Toriello V. Approach to the Genetic Evaluation of the Child with Autism. Pediatr Clin North Am. 2012; 59(1): 113-28.

41. World Health Organization. International classification of diseases (1990). Consultado: agosto de 2015. Disponible en: http://www.who.int/classifications/icd/en/bluebook. pdf?ua $=1$

42. Mannion A, Leader G. Comorbidity in autism spectrum disorder: A literature review. Research in Autism Spectrum Disorders. 2013; 7: 1595-616.

43. Mannion A, Leader G, Healy O. An investigation of comorbid psychological disorders, sleep problems, gastrointestinal symptoms and epilepsy in children and adolescents with autism spectrum disorder. Research in Autism Spectrum Disorders. 2013; 7(1): 35-42.

44. Simonoff E, Pickles A, Charman T, Chandler S, et al. Psychiatric disorders in children with autism spectrum disorders: Prevalence, comorbidity and associated factors in a population-driven sample. J Am Acad Child and Adolescent Psychiatry. 2008; 47(8), 921-9.

45. Tuchman R, Rapin I. Epilepsy in autism. The Lancet Neurolog. 2002; 1: 352-8.

46. Filipek PA. Practice parameter: screening and diagnosis of autism: report of the Quality Standards Subcommittee of the American Academy of Neurology and the Child Neurology Society. Neurology. 2000; 55(4): 468-79.

47. Johnson CP, Myers SM, Identification and evaluation of children with autism spectrum disorders. American Academy of Pediatrics Council on Children with Disabilities. Pediatrics. 2007; 120(5): 1183-206.

48. Volkmar F, Siegel M, Woodbury-Smith M, King B, McCracken J, State M. Practice parameter for the assessment and treatment of children and adolescents with autism spectrum disorder. American Academy of Child and Adolescent Psychiatry (AACAP) Committee on Quality Issues (CQI). J Am Acad Child Adolesc Psychiatry. 2014; 53(2): 237-57.

49. Ministerio de Salud y Protección Social. Instituto de Evaluación Tecnológica en Salud. Protocolo clínico para el diagnóstico, tratamiento y ruta de atención integral de niños y niñas con trastornos del espectro autista. 2015.

50. E. Baker, S.Spurling Jeste, Diagnosis and Management of Autism Spectrum Disorder in the Era of Genomics Rare Disorders Can Pave the Way for Targeted Treatments. Pediatr Clin N Am. 2015; 62: 607-18.

51. Healthcare Improvement Scotland, Scottish Intercollegiate Guideline Network. [Internet] Assessment, diagnosis and clinical interventions for children and young people with autism spectrum disorders, a national clinical guideline, number 98. Edinburgh 2007. Consultado: agosto de 2015. Disponible en: http://www.sign.ac.uk/pdf/ sign98.pdf

52. Filipek A. et al- Brain asymmetries in autism and developmental language disorder: a nested whole-brain analysis. Brain. 2005; 128: 213-26. 